\title{
Correction to: ESARA: A Framework for Enterprise Smartphone Apps Risk Assessment
}

Majid Hatamian, Sebastian Pape (D), and Kai Rannenberg

\section{Correction to:}

Chapter "ESARA: A Framework for Enterprise Smartphone Apps Risk Assessment" in: G. Dhillon et al. (Eds.): ICT Systems Security and Privacy Protection, IFIP AICT 562, https://doi.org/10.1007/978-3-030-22312-0_12

By mistake the originally published version of this chapter did not include the acknowledgement text. This has been corrected so that the updated version of the chapter now contains the following acknowledgement: This research was supported by the European Union's Horizon 2020 Research and Innovation program under the Marie Skłodowska-Curie "Privacy\&Us" project (GA No. 675730). 